\title{
ARTICLE OPEN Design of soft magnetic materials
}

\author{
Ananya Renuka Balakrishna (D) ${ }^{1 凶}$ and Richard D. James $\mathbb{D}^{2}$
}

We present a strategy for the design of ferromagnetic materials with exceptionally low magnetic hysteresis, quantified by coercivity. In this strategy, we use a micromagnetic algorithm that we have developed in previous research and which has been validated by its success in solving the "Permalloy Problem"-the well-known difficulty of predicting the composition $78.5 \% \mathrm{Ni}$ of the lowest coercivity in the Fe-Ni system - and by the insight it provides into the "Coercivity Paradox" of W. F. Brown. Unexpectedly, the design strategy predicts that cubic materials with large saturation magnetization $m_{s}$ and large magnetocrystalline anisotropy constant $\kappa_{1}$ will have low coercivity on the order of that of Permalloy, as long as the magnetostriction constants $\lambda_{100}, \lambda_{111}$ are tuned to special values. The explicit prediction for a cubic material with low coercivity is the dimensionless number $\left(c_{11}-c_{12}\right) \lambda_{100}^{2} /\left(2 \kappa_{1}\right)=81$ for $\langle 100\rangle$ easy axes. The results would seem to have broad potential application, especially to magnetic materials of interest in energy research.

npj Computational Materials (2022)8:4 ; https://doi.org/10.1038/s41524-021-00682-7

\section{INTRODUCTION}

A long-standing puzzle in materials science is understanding the origins of magnetic hysteresis in ferromagnetic materials. Hysteresis in this domain refers to the differing behaviors obtained when a demagnetized specimen is subject to an increasing magnetic field to saturation vs. that obtained when decreasing the field from saturation to zero. The effect is typically characterized by the final (absolute) value of the magnetic field after such a test, termed the coercivity. Informally, soft magnets have low coercivity. This paper is concerned with the prediction of coercivity from micromagnetic theory. An unexpected prediction of our study is that coercivity can be made very small even in materials with large magnetocrystalline anisotropy constant, as long as the magnetostrictive constants are tuned appropriately.

Aside from basic scientific interest on the origins of hysteresis and the traditional application to transformers, a strategy for the discovery of new soft magnetic materials is desirable for rapid power-conversion electronics, all-electric vehicles, and wind turbines, especially in cases where induction motors are favored. Magnetic hysteresis has also become critical to the adoption of proposed spintronic and storage devices, as requirements for limiting energy consumption have moved to the forefront ${ }^{1,2}$. These requirements impact a wide range of applications, from hand held electronic devices to storage systems and servers at data centers. While our analysis is aimed primarily at bulk applications, the key idea that micromagnetic theory with magnetostriction, together with a well-chosen potent defect, can be used to predict hysteresis suggests a strategy for the lowering of coercivity also in these small-scale applications. In fact, in certain film-based devices, the likely potent defects, such as threading dislocations in epitaxial films, are often better characterized than in bulk material.

Currently, a widely accepted strategy to lower the hysteresis in cubic ferromagnetic alloys is based on changing composition so as to reduce the magnitude of the anisotropy constant $\left|k_{1}\right|$. This has the effect of flattening the graph of magnetocrystalline anisotropy energy vs. magnetization and reducing the penalty associated with magnetization rotation. Intuitively, this makes sense, as it apparently makes available additional low-energy pathways of an alloy in a metastable state on the shoulder of the hysteresis loop, as an applied field is being lowered. A related idea is the known strategy of tuning the composition so as to be precisely at the point where two different symmetries coincide, again leading to a flattening of the magnetocrystalline anisotropy energy vs. magnetization and the lowering of hysteresis. The latter is the strategy used by Clarke and collaborators ${ }^{3,4}$ that led to the particular composition of Terfenol: $\mathrm{Tb}_{x} \mathrm{Dy}_{1-x} \mathrm{Fe}_{2}, x=0.3$. We add that modern research on these $\mathrm{RFe}_{2}$ cubic Laves phase materials has focused on the benefits of exploiting a nearby morphotropicphase boundary in these systems to enhance magnetostrictive response under small fields ${ }^{5-7}$.

However, these strategies cannot be the whole story behind coercivity. For example, in the iron-nickel system, a sharp drop in coercivity occurs at the Permalloy composition, at which $\kappa_{1}=$ $-161 \mathrm{Jm}^{-3}$. Tuning the magnetocrystalline anisotropy constant to zero in iron-nickel alloys in fact leads to an alloy composition with noticeably higher hysteresis ${ }^{8}$. Similarly, in Sendust $\left(\mathrm{Fe}_{0.85} \mathrm{~S}\right.$ $\mathrm{i}_{0.096} \mathrm{Al}_{0.054}$ alloy), the hysteresis is minimum when the magnetocrystalline anisotropy and magnetostriction constants are close to zero, and not precisely at $\kappa_{1}=0^{9}$. Finally, there are quite a few isolated examples of alloys that have very large magnetocrystalline anisotropy constants but low coercivity: an example is the uniaxial phase of $\mathrm{Ni}_{51.3} \mathrm{Mn}_{24.0} \mathrm{Ga}_{24.7}$ having a uniaxial magnetocrystalline anisotropy constant of $2.45 \times 10^{5} \mathrm{Jm}^{-3}$ and coercivity less than $1 \mathrm{kAm}^{-1}$ in single crystals ${ }^{10}$. Another example is the Galfenol alloys $\left(\mathrm{Fe}_{1-\mathrm{x}} \mathrm{Ga}_{\mathrm{x}}\right.$ for $\left.0.13 \leq x \leq 0.24\right)$ that have small magnetic hysteresis, despite their very large magnetocrystalline anisotropy constants ${ }^{11,12}$. These examples suggest that the magnetocrystalline anisotropy constant is not the only factor that governs hysteresis in magnetic alloys.

The precise role of magnetostriction constants on magnetic hysteresis is not well understood for two reasons: (a) prior research has typically ignored the contribution of magnetoelastic interactions on hysteresis, justified by the small values of the magnetostriction constants, and (b) mathematical methods, such as the linear stability analysis, overestimate the coercivity of bulk

\footnotetext{
${ }^{1}$ Aerospace and Mechanical Engineering, University of Southern California, Los Angeles, CA 90089, USA. ${ }^{2}$ Aerospace and Engineering Mechanics, University of
} Minnesota, Minneapolis, MN 55455, USA. ${ }^{\circledR}$ email: renukaba@usc.edu 
alloys-by over three orders of magnitude in some cases-as compared with measured experimental values ${ }^{13,14}$. The latter is referred to as the "Coercivity Paradox"13,15. These factors limit our understanding of how the balance between fundamental material constants-such as magnetocrystalline anisotropy and magnetostriction constants-affects magnetic coercivity.

In our recent work, we developed a computational tool based on micromagnetics, including magnetoelastic terms, that is adapted to the prediction of coercivity in bulk magnetic alloys ${ }^{16,17}$. Micromagnetics, since its first postulation in 1963 by W.F.Brown Jr. ${ }^{13}$, has been applied to a wide range of problems in ferromagnets and forms the basis to several computational frameworks ${ }^{18-20}$. Our coercivity tool is based on the micromagnetics theory ${ }^{16}$, but however differs from earlier works in the following ways: a key feature of this tool is that it uses a large but highly localized disturbance-in the form of a Néel-type spike domain-to predict magnetic coercivity. Néel spike-like domains are frequently observed to form around defects in various magnetic alloys (see ${ }^{21-25}$ for examples) and grow under an external field. In the absence of this spike-domain, the second variation of the micromagnetic energy misses the energy barrier for magnetization reversal ${ }^{13,26}$. Instead, we model a large localized disturbance (i.e., compute a nonlinear stability analysis) to predict coercivity on the shoulder of the hysteresis curve, see Fig. 1. Other features of our coercivity tool are that we account for magnetoelastic interactions, however small, in estimating coercivity of bulk magnetic alloys; and we use the ellipsoid and reciprocal theorems to accelerate our coercivity calculations, see ${ }^{16}$. Our computations are fully three-dimensional with the magnetization evolving from (010) to (100) in all directions as one leaves the spike domain.

To arrive at the Néel spike as a reasonable description of a potent defect, we include in ${ }^{16}$ a study of alternative nuclei, including rotated spikes and multiple spikes. These spike domains serve as nuclei that grow during magnetization reversal. In this previous study ${ }^{16}$, we investigated the role of defect geometry, defect orientation, and defect number on magnetic coercivity, and we found that these features did not significantly affect coercivity when compared with fundamental material constants. Mathematically, they can be interpreted as localized disturbances that, under the right combination of material constants and suitable applied field, are able to surmount an energy barrier ${ }^{27-30}$ Small, smooth disturbances of a homogeneous state are not able to surmount this barrier at realistic applied fields due to the dominance of domain-wall energy at small scales, and, in our view, this is the essence of the Coercivity Paradox ${ }^{16,17}$. Mathematically, the situation is that the study of the second variation of the micromagnetic energy misses the important energy barrier, while it is captured as a large-amplitude, but localized, disturbance.

Our viewpoint is consistent with the thesis of Pilet $^{27}$ who examines the microscopic state on the shoulder of the hysteresis loop (far away from where coercivity is measured) using magnetic force microscopy, and finds good correlation with the presence of large localized disturbances. Another feature of our tool is that we account for the magnetoelastic interactions-however small-in estimating coercivity. Our results are in a form that is amenable to alloy development, as in related searches for low hysteresis-phase transformations ${ }^{31}$.

An alternative highly effective route to low hysteresis in magnetic materials is the synthesis of nanocrystalline material ${ }^{32}$. For example, the powder synthesis and rapid solidification techniques have resulted in nanocrystalline and amorphous magnetic alloys that offer low hysteresis and enhanced permeability $2,33,34$. Here we do not specifically analyze this situation, but we think this should be possible in the nanocrystalline case, though challenging due to the many grains that would likely have to be considered.

The aim of the present work is to identify combinations of material constants, including saturation magnetization $\left(m_{s}\right)$, magnetocrystalline anisotropy constant $\left(\kappa_{1}\right)$, elastic moduli $\left(c_{11}\right.$, $\left.c_{12}, c_{44}\right)$, and magnetostriction constants $\left(\lambda_{100}, \lambda_{111}\right)$ that give the lowest coercivity in a cubic material. We do not vary the exchange constant, which in practice does not vary much: we also give arguments why, when combined with the demagnetization energy, it should matter little. We find the striking result that magnetostriction plays a critical role, and more importantly, coercivities on the order of those found in very soft materials such as Permalloy are predicted to be possible in materials with large magnetocrystalline anisotropy constant, as long as the magnetostriction constants are tuned to special values. We find that magnetocrystalline anisotropy and magnetostriction constants play a particularly important role, but neither has to be small.

The simulations are carried out using our newly developed coercivity tool. Details on our micromagnetic algorithm are described in "Methods". Specifically, we apply this tool in two studies: in Study 1, we test the hypothesis that the tuning of (a)

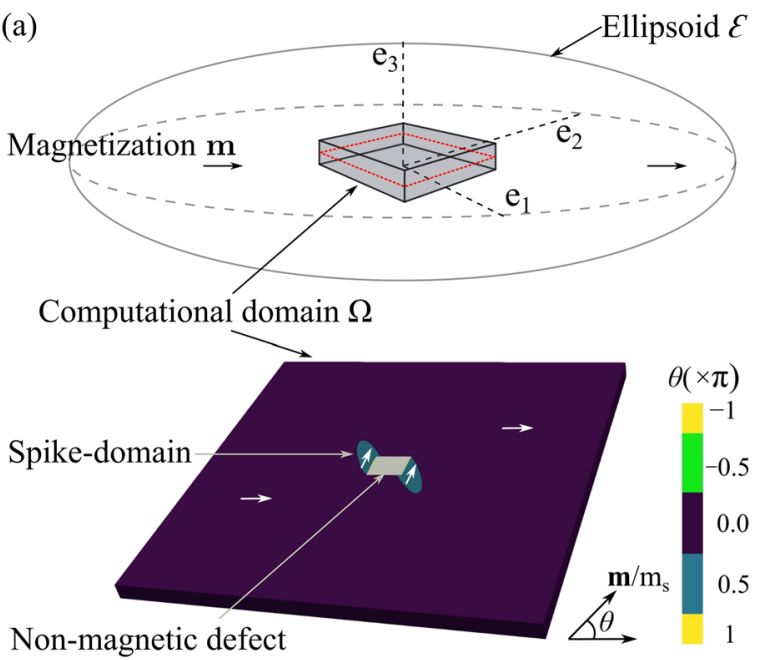

(b) Spike-domains are commonly imaged in experiments
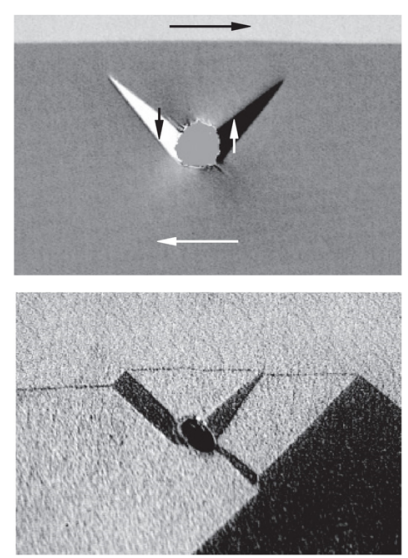

Fig. 1 We model a 3D computational domain with a spike-domain microstructure. a The 3D computational domain is $\Omega$ embedded inside an oblate ellipsoid $\mathcal{E}$, which is several times larger in size than $\Omega$. A spike-domain microstructure forms around a nonmagnetic defect that grows under an applied field. (b) Spike-domain microstructures are commonly found in magnetic materials around defects such as cavities and pores in Fe film and Fe-Si crystal, respectively ${ }^{21,40}$. (Reprinted subfigure (b) with permission from ref. ${ }^{40}$. Copyright (2020) by the American Physical Society). 
magnetostriction constants, in addition to the magnetocrystalline anisotropy constant, is necessary to reduce coercivity in magnetic alloys. Here we study how combinations of $\kappa_{1}$ and $\lambda_{100}$ affect coercivity, and ignore the contribution from $\lambda_{111}=0$. In total, we compute coercivity values from $N=2,163$ independent simulations. In Study 2, we test our hypothesis that there exists a specific combination of material constants $-\kappa_{1}, \lambda_{100}, \lambda_{111}$-at which magnetic coercivity is the lowest. Here, we compute coercivity by systematically varying the magnetocrystalline anisotropy and the magnetostriction constants, in the ranges $0 \leq \kappa_{1} \leq 2000 \mathrm{~J} \mathrm{~m}^{-3}$, $-2000 \mu \epsilon \leq \lambda_{100} \leq 2000 \mu \epsilon$, and $0 \leq \lambda_{111} \leq 600 \mu \epsilon$, respectively $(N=$ $605)$. Overall, our computations from over 2500 independent simulations show that the lowest coercivity is attained when the dimensionless number $\frac{\left(c_{11}-c_{12}\right) \lambda_{100}^{2}}{2 \kappa_{1}} \approx 81$. Here, $c_{11}, c_{12}$ are the elastic stiffness constants of the soft magnet assuming a linear cubic relation. To our knowledge, this discovery of the balance between material constants at which magnetic hysteresis is small has not been proposed before. A theoretical analysis supporting the importance of this dimensionless number is given below. This analysis further supports the idea that, in other situations, a second dimensionless number $\frac{c_{12} \lambda_{100}^{2}}{2 \kappa_{1}}$ may become important, and tuning the stiffness constants $c_{11}$ and $c_{12}$, so that both of these dimensionless constants have particular values may be desirable ${ }^{35}$.

\section{RESULTS}

\section{Computation of coercivity}

In Study 1, we test our hypothesis that the magnetostriction constants, in addition to the magnetocrystalline anisotropy constant, are necessary to reduce coercivity in magnetic alloys. Figure 2(a) shows a heat map of coercivity values as a function of the magnetocrystalline anisotropy constant $\kappa_{1}$ and the

(a) Coercivity map

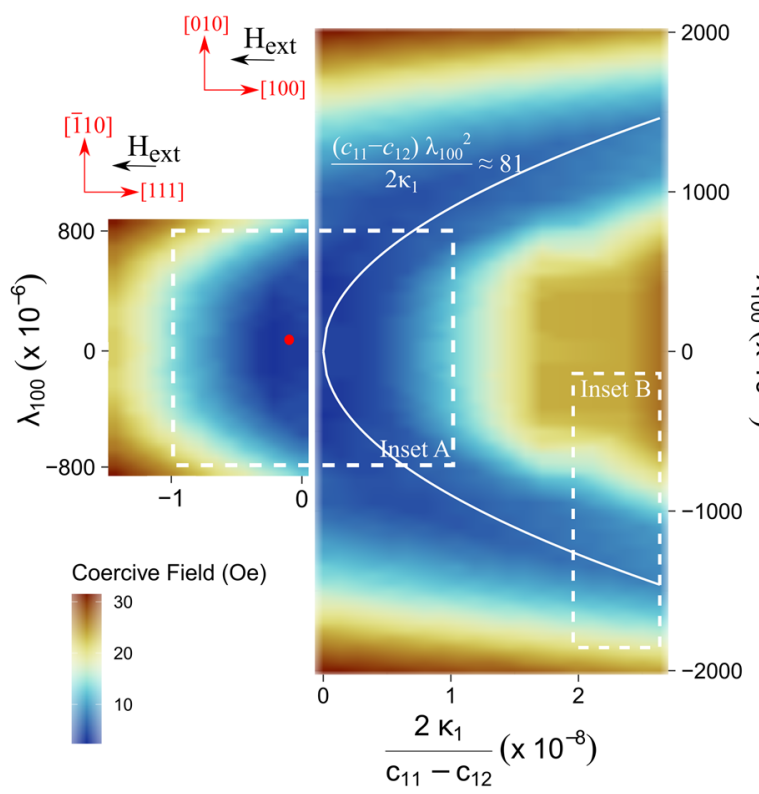

magnetostriction constant $\lambda_{100}$. A key feature of this plot is that the coercivity is minimum along a curve described by $\frac{\left(c_{11}-c_{12}\right) \lambda_{100}^{2}}{2 \kappa_{1}} \approx 81$. As expected, the coercivity is small when $\kappa_{1} \rightarrow 0$ and the magnetostriction constant is small $\lambda_{100} \rightarrow 0$, see Inset $A$. However, surprisingly, we find that the coercivity value is also small for non-zero magnetocrystalline anisotropy constants $\kappa_{1} \gg$ 0 with suitable combinations of the magnetostriction constant, see Inset B. Note the rather large magnetocrystalline anisotropy constants being considered, well outside the range associated with normal soft magnetism. Furthermore, the minimum coercivity valleys are symmetric about the $\lambda_{100}=0$ axis. This symmetric response arises from the even terms $\lambda_{100}^{2}$ in the free-energy expression. We observe a similar lowering of coercivity in magnetic alloys with $\kappa_{1}<0$ at suitable values of $\lambda_{111}$ magnetostriction constant (see Supplementary Fig. 2).

Figure $2(b-c)$ are $3 D$ surface plots of the inset regions " $A$ " and " $B$ " respectively. The "wells" in these plots correspond to combinations of material constants at which coercivity is minimum. Figure 2(b) is a $3 \mathrm{D}$ plot of the inset region " $\mathrm{A}$ "-here, we note a discontinuity or a jump in coercivity values at $\kappa_{1}=0$. This discontinuity is because of the change in easy axes for magnetic alloys with $\kappa_{1}>0$ and $\kappa_{1}<0$. For example, we compute coercivities along [100] and [111] crystallographic directions for magnetic alloys with $\kappa_{1}>0$ and $\kappa_{1}<0$, respectively. These alloys have different values of magnetostriction constants along their easy axes. We believe that the computed rapid change of coercivity at $\kappa_{1}=0$ is real, and arises from the anisotropy of these magnetostriction constants. Figure 2(c) shows the 3D surface plot of the inset region $\mathrm{B}$. Here, coercivities comparable to that of the permalloy composition are achieved at large magnetocrystalline anisotropy values $\kappa_{1} \approx 1700 \mathrm{~J} \mathrm{~m}^{-3}$ with suitable combinations of the magnetostriction constant $\lambda_{100} \approx 1200 \mu \epsilon$.

(b) Inset $\mathrm{A}$

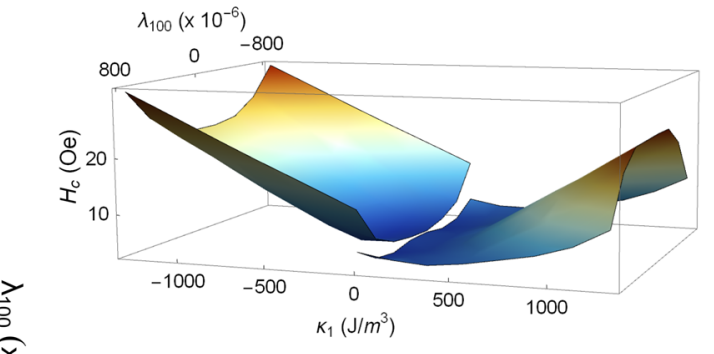

(c) Inset B

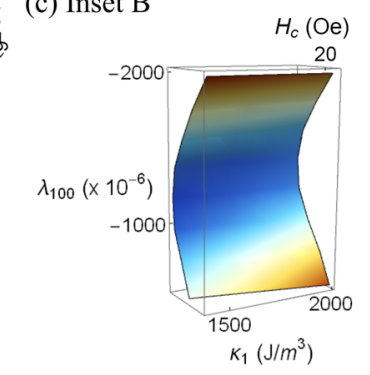

Fig. 2 The coercivity map as a function of the magnetocrystalline anisotropy $\kappa_{1}$ and the magnetostriction constant $\lambda_{100}$ a We carried out a total of $N=2163$ computations with the magnetostriction constant $\lambda_{111}$ set to zero. We find that minimum coercivity is achieved when $\frac{\left(c_{11}-c_{12}\right) \lambda_{100}^{2}}{2 K_{1}} \approx 81$. For comparison, the solid red dot corresponds to the coercivity of the permalloy composition. Note, the parabolic relationship is a best-fit polynomial to the spread of coercivity data from our calculations. Alloys, such as the permalloy, lie close to the vertex of this parabola and we interpret this as the shortest distance to the parabola with a dimensionless constant of 81 . The axes (in red) indicate the crystallographic directions along which coercivity values were measured for alloys with $\kappa_{1}>0$ and $k_{1}<0$, respectively. The 3D surface contours of the coercivity values in (b) "inset $A$ " and (c) "inset B" are shown. These plots show coercivity wells (regions of minimum coercivity) as $k_{1} \rightarrow 0$ and $\kappa_{1}>>0$, respectively. Note, in subfigure (b), the 3D surface contour is discontinuous along $\kappa_{1}=0$-we attribute this discontinuity to the transformation of easy axes from [100] to [111] crystallographic direction. 
Overall, the findings from Study 1 contradict the general understanding of hysteresis in magnetism, i.e., the magnetocrystalline anisotropy constant needs to be near zero for small hysteresis. Our findings show that magnetic hysteresis (or coercivity value) is small not only when $\kappa_{1} \rightarrow 0$ but also when $\kappa_{1}>>0$, given suitable values of the magnetostriction constants. Figure 2 shows that the magnetostriction constant, $\lambda_{100}$, in addition to the magnetocrystalline anisotropy constant $\kappa_{1}$ plays an important role in reducing coercivity in magnetic alloys.

Thus far, we investigated coercivity values by setting one of the magnetostriction constants $\lambda_{111}$ to be zero. In Study 2, we test the hypothesis that there exists a specific combination of material constants, namely $\kappa_{1}, \lambda_{100}, \lambda_{111}$, at which magnetic coercivity is the lowest. We compute coercivities at every combination of $\kappa_{1}, \lambda_{100}$, and $\lambda_{111}$ in the parameter space $0 \leq \kappa_{1} \leq 2000 \mathrm{~J} \mathrm{~m}^{-3}$ and $-2000 \mu \epsilon \leq \lambda_{100} \leq 2000 \mu \epsilon$ and $0 \leq \lambda_{111} \leq 600 \mu \epsilon$.

Figure $3(\mathrm{a})$ shows the coercivity map as a function of $\kappa_{1}$ and $\lambda_{100}$ for increasing values of $\lambda_{111}$. A key feature here is that the minimum coercivity relationship $\kappa_{1} \propto \lambda_{100}^{2}$ is unique at each value of the $\lambda_{111}$ magnetostriction constant. For example, the minimum coercivity valleys gradually widen and become asymmetric about $\lambda_{100}=0$, with increasing value of the $\lambda_{111}$ magnetostriction constant. We identify combinations of material constants at which minimum coercivity is achieved, and then plot a 3D surface through these points in the $\kappa_{1}-\lambda_{100}-\lambda_{111}$ parameter space, see Fig. 3(b). This 3D plot represents a surface through the material parameter space on which coercivity is small.

\section{Theoretical analysis}

The results of our studies above can be understood in the following way. We begin from the free energy that we have used in the simulations of micromagnetics (see Figs. 4, 5) in dimensional form ${ }^{16}$,

$$
\begin{aligned}
& \int_{\Omega}\left\{\nabla \mathbf{m} \cdot \mathrm{A} \nabla \mathbf{m}+\kappa_{1}\left(\mathrm{~m}_{1}^{2} \mathrm{~m}_{2}^{2}+\mathrm{m}_{2}^{2} \mathrm{~m}_{3}^{2}+\mathrm{m}_{3}^{2} \mathrm{~m}_{1}^{2}\right)\right. \\
&\left.\quad+\frac{1}{2}\left[\mathbf{E}-\mathbf{E}_{\mathbf{0}}(\mathbf{m})\right] \cdot \mathbb{C}\left[\mathbf{E}-\mathbf{E}_{\mathbf{0}}(\mathbf{m})\right]-\mu_{0} m_{s} \mathbf{H}_{\mathrm{ext}} \cdot \mathbf{m}\right\} \mathrm{d} \mathbf{x}+\frac{1}{2} \int_{\mathbb{R}^{3}} \mu_{0}\left|\mathbf{H}_{\mathbf{d}}\right|^{2} \mathrm{~d} \mathbf{x}
\end{aligned}
$$

where $\mathbf{E}=\frac{1}{2}\left(\nabla \mathbf{u}+(\nabla \mathbf{u})^{T}\right)$ and the energy is to be minimized, or minimized locally, over the pair of functions $\mathbf{u}, \mathbf{m} \in H^{1}(\Omega)$. Here, $\mathbf{m}$ (with components on the cubic axes $m_{1}, m_{2}, m_{3}$ ) has been previously nondimensionalized so $\mathbf{m} \cdot \mathbf{m}=1$, and the demagnetization field satisfies the magnetostatic equations $\mathbf{H}_{\mathbf{d}}=-\nabla \zeta_{\mathbf{m}}$, $\nabla \cdot\left(-\nabla \zeta_{\mathbf{m}}+m_{s} \mathbf{m}\right)=0$ on all of space, so $\mathbf{H}_{\mathbf{d}}$ has the dimensions of $m_{s}$ as does $\mathbf{H}_{\text {ext }}$. Please note that $\mathbf{m}$ is extended to $\mathbb{R}^{3}$ by making it vanish outside $\Omega$. Typical accepted values from a large compositional space appropriate to Fig. 3(a) including most of the $\mathrm{Fe}-\mathrm{Ni}$ system, are

$$
\begin{aligned}
\mathrm{A} \sim & 10^{-11} \mathrm{Jm}^{-1}, \kappa_{1} \sim 0-6 \times 10^{3} \mathrm{Nm}^{-2}, \lambda_{100} \sim 0-2000 \times 10^{-6}, \lambda_{111} \sim 0-600 \times 10^{-6}, \\
& m_{s} \sim 10^{6} \mathrm{Am}^{-1}, \mu_{0} \sim 1.3 \times 10^{-6} \mathrm{NA}^{-2}, c_{11}, c_{12}, c_{44} \sim 10-24 \times 10^{10} \mathrm{Nm}^{-2}
\end{aligned}
$$

A nondimensional form is obtained by dividing the micromagnetic energy by $\mu_{0} m_{s}^{2} \sim 10^{6} \mathrm{Nm}^{-2}$, changing variables $\mathbf{x}^{\prime}=\frac{1}{\ell} \mathbf{x} \in \Omega^{\prime}$, where $\ell$ is a typical length scale and $\mathbf{x}^{\prime}$ is dimensionless. This gives a typical nondimensional value of the micromagnetic coefficients

$$
\begin{gathered}
\frac{A}{\ell^{2} \mu_{0} m_{s}^{2}} \sim 10^{-17}, \frac{\kappa_{1}}{\mu_{0} m_{s}^{2}} \sim 10^{-3}, \frac{\left(c_{11}-c_{12} \text { or } c_{44}\right)\left(\lambda_{100}^{2} \text { or } \lambda_{111}^{2}\right)}{\mu_{0} m_{s}^{2}} \sim 10^{-5}-10^{-1}, \\
\frac{1}{m_{s}} H_{\text {ext }} \sim 10^{-3}, \frac{1}{m_{s}^{2}} H_{\mathrm{d}}^{2} \sim 1
\end{gathered}
$$

The range of magnetostrictive coefficients is consistent with Fig. 3 (a), and for material constants with a size range, we choose a typical intermediate value. A caveat with these numbers is the observation made by Brown ${ }^{26}$ (discussed also $\mathrm{in}^{21}$ ) that the formal linearization of geometrically nonlinear micromagnetics that gives Eq. (1) implies a possible $\mathbf{m}$ dependence of the elastic moduli $c_{11}$, $c_{12}, c_{44}$. This dependence is usually neglected, as is done here. Note from Eq. (3) the wide ranging values of magnetostrictive energy and its relative importance generally.

It is seen from the nondimensionalized coefficient Eq. (3) that magnetostriction and demagnetization energy are dominant, but it is important to observe that each can be made negligible by suitable magnetization distributions. The magnetostrictive energy (a)

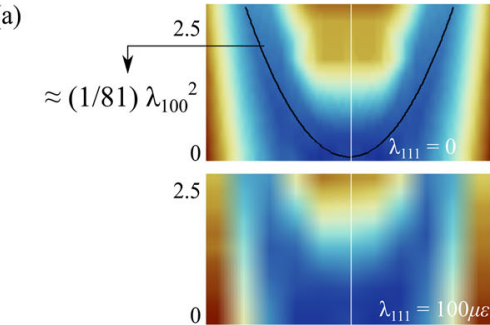

$\frac{2 \kappa_{1}}{c_{11}-c_{12}}\left(\times 10^{-8}\right)$

2.5

(b)
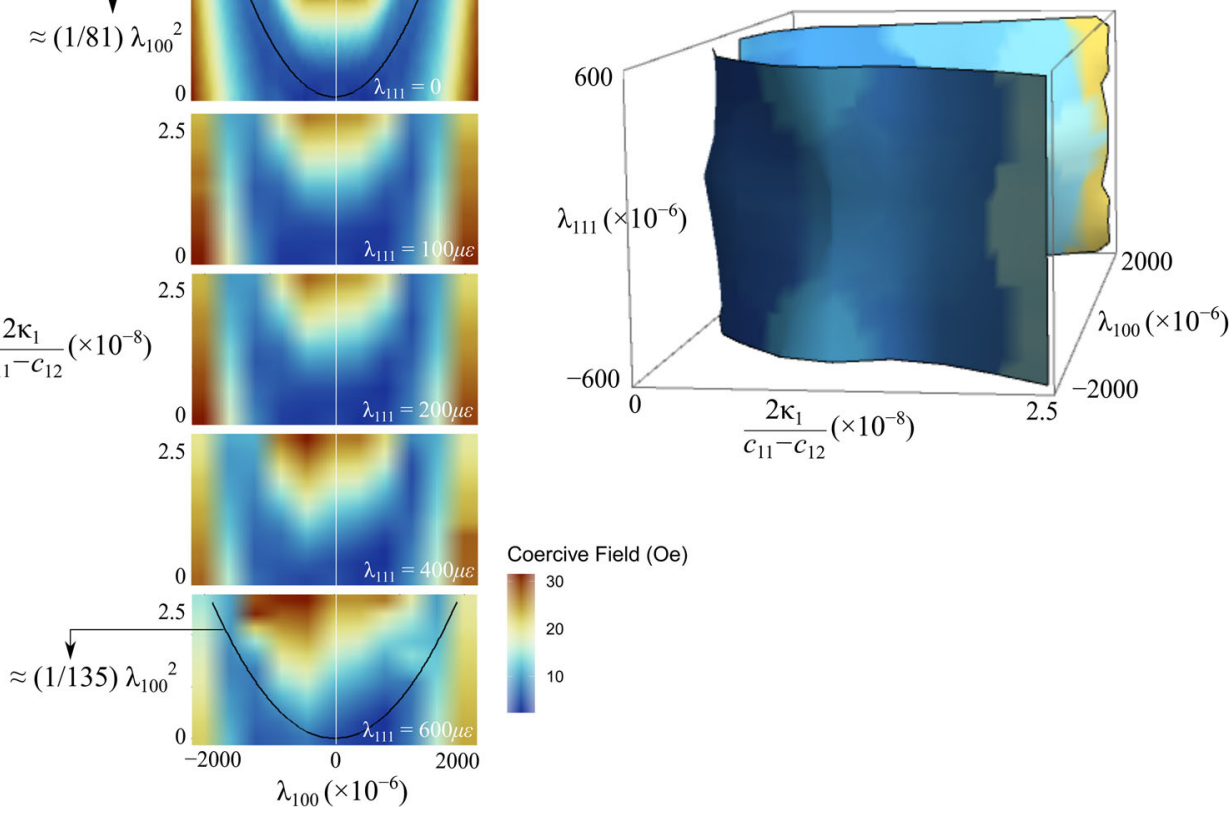

Fig. 3 The coercivity map as a function $\kappa_{\mathbf{1}}, \boldsymbol{\lambda}_{\mathbf{1 0 0}}$, and $\boldsymbol{\lambda}_{\mathbf{1 1 1}}$. a The minimum coercivity is achieved along a parabolic relation, $\frac{\left(c_{11}-c_{12}\right) \lambda_{100}^{2}}{2 \kappa_{1}} \approx p$. Here, the value of coefficient $p$ depends on the value of the second magnetostriction constant $\lambda_{111}$. For example, as $\lambda_{111}$ increases and the coercivity well loses symmetry across the $\lambda_{100}=0$ axes. b The 3D surface plot identifies a combination of material constants, namely $\kappa_{1}, \lambda_{100}$, and $\lambda_{111}$, at which the coercivity is small. 
can be made to vanish by choosing a magnetization that satisfies $\operatorname{curl}\left(\operatorname{curl} \mathbf{E}_{0}(\mathbf{m})\right)^{T}=0$, that is, $\mathbf{E}_{0}(\mathbf{m}(\mathbf{x}))=\frac{1}{2}\left(\nabla \mathbf{u}+(\nabla \mathbf{u})^{T}\right)$ is the symmetric part of a gradient, while the demagnetization energy vanishes on divergence-free magnetizations. In addition, we note that the exchange energy is one of the smallest energy contributions and its contribution further decreases at increasing length scales. Although the exchange constant could be affected by temperature, composition, and presence of defects, its order of magnitude $A \approx 10^{-11} \mathrm{~J} \mathrm{~m}^{-1}$ does not significantly affect large-scale micromagnetic simulations. Consequently, we do not vary the exchange constant in our calculations.

We first explain from a theoretical viewpoint why the Néel spike attached to a defect of the type we have chosen is a particularly potent perturbation. A key observation comes from symmetry and holds also in the more general case of a geometrically nonlinear magnetoelastic free energy (see Section 6 of $^{36}$ ). The observation is that domain walls involving a jump in the magnetization $\mathbf{m}^{+}-\mathbf{m}^{-} \neq 0$, where $\mathbf{m}^{+}$and $\mathbf{m}^{-}$minimize the magnetocrystalline anisotropy energy density and satisfy the divergence-free condition $\left(\mathbf{m}^{+}-\mathbf{m}^{-}\right) \cdot \mathbf{n}=0$ at an interface with normal $\mathbf{n}$, have the property that they give rise to strains that are perfectly mechanically compatible in the sense that $\mathbf{E}_{0}\left(\mathbf{m}^{+}\right)-$ $\mathbf{E}_{0}\left(\mathbf{m}^{-}\right)=\frac{1}{2}(\mathbf{a} \otimes \mathbf{n}+\mathbf{n} \otimes \mathbf{a})$ for some vector $\mathbf{a}$. The latter is the jump condition implying the existence of a continuous displacement across the interface. These conditions hold also for typical domain-wall models with remote boundary conditions given by $\left(\mathbf{m}^{+}, \mathbf{E}_{0}\left(\mathbf{m}^{+}\right)\right)$and $\left(\mathbf{m}^{-}, \mathbf{E}_{0}\left(\mathbf{m}^{-}\right)\right)$. This argument applies not only to materials with cubic symmetry but also to many lower-symmetry systems (see ${ }^{36}$ for the precise statement of this result).

To understand the relation between this symmetry argument and the Néel spike, we substitute the form of $\mathbb{C}$ and $\mathbf{E}_{0}(\mathbf{m})$ for cubic materials into Eq. (1). The latter is

$\mathbf{E}_{0}(\mathbf{m})=\frac{3}{2}\left(\begin{array}{lll}\lambda_{100}\left(\mathrm{~m}_{1}^{2}-\frac{1}{3}\right) & \lambda_{111} \mathrm{~m}_{1} \mathrm{~m}_{2} & \lambda_{111} \mathrm{~m}_{1} \mathrm{~m}_{3} \\ \lambda_{111} \mathrm{~m}_{1} \mathrm{~m}_{2} & \lambda_{100}\left(\mathrm{~m}_{2}^{2}-\frac{1}{3}\right) & \lambda_{111} \mathrm{~m}_{2} \mathrm{~m}_{3} \\ \lambda_{111} \mathrm{~m}_{1} \mathrm{~m}_{3} & \lambda_{111} \mathrm{~m}_{2} \mathrm{~m}_{3} & \lambda_{100}\left(\mathrm{~m}_{3}^{2}-\frac{1}{3}\right)\end{array}\right)$

in the orthonormal cubic basis. We consider only the case $\kappa_{1}>0$ corresponding to $\langle 100\rangle$ easy axes, for which we have the most data above. The case $\kappa_{1}<0$ corresponding to $\langle 111\rangle$ easy axes is handled similarly. Without loss of generality, we also divide the whole energy by the dimensionless number $\kappa_{1} / \mu_{0} m_{s}^{2}$. In addition, we define a new displacement $\hat{\mathbf{u}}(\mathbf{x})$ by $\mathbf{u}(\mathbf{x})=\lambda_{100} \hat{\mathbf{u}}(\mathbf{x})$ with the corresponding strain tensor $\mathbf{E}=\lambda_{100} \mathbf{E}$. Minimization of energy using $\mathbf{u}$ is equivalent to the same using $\hat{\mathbf{u}}$, and this equivalence applies also to local minimization or the relative height of an energy barrier. These changes give the explicit nondimensional form of the free energy

$$
\begin{aligned}
& \int_{\Omega^{\prime}} \frac{A}{e^{2} K_{1}} m_{i, j} m_{i, j}+\left(m_{1}^{2} m_{2}^{2}+m_{2}^{2} m_{3}^{2}+m_{3}^{2} m_{1}^{2}\right)
\end{aligned}
$$

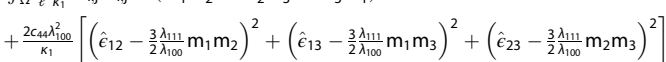

$$
\begin{aligned}
& +\left(\frac{\left(c_{11}-c_{12}\right) \lambda_{100}^{2}}{2 k_{1}}\right)\left[\left(\hat{\epsilon}_{11}-\frac{3}{2}\left(m_{1}^{2}-\frac{1}{3}\right)\right)^{2}+\left(\hat{\epsilon}_{22}-\frac{3}{2}\left(m_{2}^{2}-\frac{1}{3}\right)\right)^{2}+\left(\hat{\epsilon}_{33}-\frac{3}{2}\left(m_{3}^{2}-\frac{1}{3}\right)\right)^{2}\right] \\
& +\frac{c_{12} \lambda_{100}^{2}}{2 k_{1}}\left(\hat{\epsilon}_{11}+\hat{\epsilon}_{22}+\hat{\epsilon}_{33}\right)^{2}-\frac{\mu_{0} m_{5}}{\kappa_{1}}\left(H_{\mathrm{e} 1} \mathrm{~m}_{1}+H_{\mathrm{e} 2} \mathrm{~m}_{2}+H_{\mathrm{e} 3} \mathrm{~m}_{3}\right)-\frac{\mu_{0} m_{\mathrm{s}}}{2 \mathrm{~K}_{1}}\left(H_{\mathrm{d} 1} \mathrm{~m}_{1}+H_{\mathrm{d} 2} \mathrm{~m}_{2}+H_{\mathrm{d} 3} \mathrm{~m}_{3}\right) \mathrm{d} \mathbf{x},
\end{aligned}
$$

where we have used the magnetostatic equation to include the demagnetization term in the integrand. Also, $\hat{\epsilon}_{i j}=\frac{1}{2}\left(\hat{u}_{i, j}+\hat{u}_{j, i}\right)$, and for simplicity, we have dropped the prime on $\mathbf{x}$. However, $\Omega^{\prime}$ remains dimensionless.

Now, we make an observation about the structure of Eq. (5) relating to the symmetry argument given above. With $\kappa_{1}>0$ as assumed, the two magnetizations $\mathbf{m}^{-}=(1,0,0)$ and $\mathbf{m}^{+}=(0,1,0)$ satisfy $\left(\mathbf{m}^{+}-\mathbf{m}^{-}\right) \cdot \mathbf{n}=0$, where $\mathbf{n}=\frac{1}{\sqrt{2}}(1,1,0)$. Therefore, by the symmetry argument described above, $\mathbf{E}_{0}\left(\mathbf{m}^{+}\right)-\mathbf{E}_{0}\left(\mathbf{m}^{-}\right)=\frac{1}{2}(\mathbf{a} \otimes$ $\mathbf{n}+\mathbf{n} \otimes \mathbf{a})$ and it is indeed verified that that this holds with $\mathbf{a}=\frac{3 \sqrt{2} \lambda_{100}}{2}(-1,1,0)$. The values of the strains are given in Fig. $6(\mathrm{c})$.
Importantly, these choices of $\mathbf{m}^{+}, \mathbf{E}_{0}\left(\mathbf{m}^{+}\right)$, and $\mathbf{m}^{-}, \mathbf{E}_{0}\left(\mathbf{m}^{-}\right)$make the magnetocrystalline anisotropy energy and all three magnetostrictive terms in Eq. (5) vanish, and also give a locally divergence-free magnetization. Recalling that the simulations are 3D (see Fig. 5), note that Fig. 6(c) can be confined to a slab between two surfaces parallel to the plane of the page and, if $\mathbf{m}$ $(\mathbf{x})=\mathbf{m}^{-}$is assigned outside the slab, then these surfaces are also pole-free. These facts support the potency (i.e., low-energy barrier) provided by the Néel spikes.

At the transient state, the magnetic poles on the tips of the spike domains are far apart and the geometric features of the spike domain have evolved to compatible interfaces. Similar symmetry arguments can be made for transient-state microstructures with multiple Néel spikes and/or other defect geometries. In these cases, the magnetic material constants dominate the energy barrier, and the defect geometries appear to play a negligible role.

Thus, we arrive at the following scenario typical of nucleation. When the applied field is large, the magnetocrystalline anisotropy energy of a large spike is disfavored, and spike is small and collapsed near the defect, due to the dominating influence of (diffuse) domain-wall energy at small scales. As the applied field is decreased, the magnetocrystalline anisotropy and demagnetization energies favor the growth of the spike. A local energy maximum is reached, beyond which an energydecreasing path is possible, leading to complete reversal of the magnetization.

The parabolic form of the locus of the lowest coercivity points in Figs. 3(a) and 2(a) can now be understood heuristically. The terms involving the very small nondimensional exchange constant and very large multiplier of the nondimensional demagnetization energy are the only terms that involve $\nabla \mathbf{m}$. These are expected to compete within the diffuse domain walls present in these simulations, but otherwise lead only to a neardivergence-free magnetization, as suggested by the inequality of arithmetic-geometric means in the form

$$
\left(\sqrt{\frac{A}{\ell^{2} \kappa_{1}}} \mathrm{~m}_{\mathrm{i}, \mathrm{j}}-\sqrt{\frac{\mu_{0}}{6 \kappa_{1}}}\left|\nabla \zeta_{\mathbf{m}}\right| \delta_{i j}\right)\left(\sqrt{\frac{A}{\ell^{2} \kappa_{1}}} \mathrm{~m}_{\mathrm{i}, \mathrm{j}}-\sqrt{\frac{\mu_{0}}{6 \kappa_{1}}}\left|\nabla \zeta_{\mathbf{m}}\right| \delta_{i j}\right) \geq 0,
$$

which implies, using the magnetostatic equations $\mathbf{H}_{\mathbf{d}}=-\nabla \zeta_{\mathbf{m}}$ and $\nabla \cdot\left(-\nabla \zeta_{\mathbf{m}}+m_{s} \mathbf{m}\right)=0$, that

$$
\frac{A}{\ell^{2} \kappa_{1}} \mathrm{~m}_{\mathrm{i}, \mathrm{j}} \mathrm{m}_{\mathrm{i}, \mathrm{j}}-\frac{\mu_{0} \mathrm{~m}_{\mathrm{s}}}{2 \kappa_{1}} \mathbf{H}_{\mathbf{d}} \cdot \mathbf{m} \geq 2 \sqrt{\frac{\mathrm{A} \mu_{0}}{6 \ell^{2} \kappa_{1}^{2}}}(\operatorname{div} \mathbf{m})\left|\nabla \zeta_{\mathbf{m}}\right| .
$$

In the geometry being considered, the heuristic argument for the energy barrier suggests that the shear strains in this geometry play a minor role, and this is supported by typical measurements of the shear strains across the spike, Fig. $7(a-c)$, taken from the simulations. This observation, together with the fact that the magnetization is near the easy axes [100] or [010] in the region of the spikes (so that $m_{i} m_{j} \approx 0, i \neq j$ ), indicates that the constant $c_{44} \lambda_{100}{ }^{2} / \kappa_{1}$ will play only a minor role in determining the energy barrier.

Granted these approximations, the height of the energy barrier must then be affected mainly by the remaining dimensionless constants

$$
\frac{\left(c_{11}-c_{12}\right) \lambda_{100}^{2}}{2 \kappa_{1}} \text { and } \frac{c_{12} \lambda_{100}^{2}}{2 \kappa_{1}} \text {. }
$$

Again, from the simulations (Fig. $7(d-f))$ tr $\mathbf{E}$ is quite small in comparison with the multiplier of $\left(c_{11}-c_{12}\right) \lambda_{100}^{2} /\left(2 \kappa_{1}\right)$, and so we expect the latter, $\left(c_{11}-c_{12}\right) \lambda_{100}^{2} /\left(2 \kappa_{1}\right)$, to be the key dimensionless constant that governs the height of the barrier. In simulations, the term containing this constant accounted for up to $80 \%$ of the total magnetoelastic energy. 
The value of this dimensionless constant giving the lowest energy barrier indicated by the simulations is

$\frac{\left(c_{11}-c_{12}\right) \lambda_{100}^{2}}{2 \kappa_{1}}=81.0056$

As we have noticed previously ${ }^{17}, 78.5 \%$ Ni Permalloy does not exactly fall on the computed parabola given by Eq. (9). This may indicate that there are opportunities for lowering the coercivity of permalloy. It is possible that favorable heat treatments of permalloy do just that by modifying the material constants present in Eq. (9). An alternative possibility is that, while Eq. (9) may be the primary dimensionless constant affecting coercivity, both constants given in Eq. (8) may play a role, indicating that the fine-tuning of elastic moduli according to their relative roles in the two constants of Eq. (8) may be a route to even lower coercivity.

\section{DISCUSSION}

At present, the conventional approach to develop soft magnets is to reduce the magnetocrystalline anisotropy value to zero. Consequently, the search for soft magnets is concentrated around the $\kappa_{1} \rightarrow 0$ region. Our theory-guided prediction suggests that in addition to the coercivity well at $\kappa_{1} \rightarrow 0$ region, there exists other regions along $\frac{\left(c_{11}-c_{12}\right) \lambda_{100}^{2}}{2 k_{1}} \approx 81$ at which coercivity is small. This analytical relation between material constants gives greater freedom for alloy development and increases the parameter space to discover novel soft magnets. In summary, our findings show that the magnetostriction constants, in addition to the magnetocrystalline anisotropy constant, play an important role in governing magnetic coercivity. This was the case in study 1 when coercivity was minimum along a parabolic relation given by $\frac{\left(c_{11}-c_{12}\right) \lambda_{100}^{2}}{2 k_{1}} \approx 81$ with $\lambda_{111}=0$ in magnetic alloys. In study 2 , we identified a $3 \mathrm{D}$ surface topology on which the coercivity is small. Below, we discuss some limitations of our findings and then present its potential impact to the magnetic alloy development program.

Two features of this work limit the conclusions we can draw about the fundamental relationship between magnetic material constants. First, we compute coercivities assuming a simple defect structure (i.e., two spike domains formed around a nonmagnetic inclusion) in an oblate ellipsoid. While defect geometry has been shown to have surprisingly small effect on the coercivity values ${ }^{16}$, it is not known whether and how different defect geometries, and crystallographic texture of the magnetic alloy would affect our predictions of the parabolic relation for minimum coercivity. Second, the presence of mechanical loads, such as residual strains or boundary loads, could affect the delicate balance between the magnetic material constants. The idealized stress-free conditions in our model are subject to the shortcomings associated with the presence of microstructural inhomogeneities and surface conditions in bulk materials. Whether accounting for these inhomogeneities would yield comparable results in experiments is an open question. With these reservations in mind, we next discuss the impact of our findings to the alloy-development program.

A key feature of our work is that we demonstrate that the magnetostriction constants play an important role in governing magnetic coercivity. These findings contrast with prior research in which the magnetocrystalline anisotropy constant has been regarded as the only material parameter that governs magnetic hysteresis, and the contribution from magnetostriction constants has been largely neglected. Consequently, the commonly accepted norm in the literature is that magnetic alloys with small magnetocrystalline anisotropy constant have small coercivity, and magnetic alloys with large magnetocrystalline anisotropy constant have large coercivity. However, by accounting for both magnetocrystalline anisotropy and magnetostriction constants, we show that magnetic alloys, despite their large $\kappa_{1}$ values, have small coercivities at specific combinations of the magnetostriction constants.

Another significant feature of our work is that we identify a relationship $\frac{\left(c_{11}-c_{12}\right) \lambda_{100}^{2}}{2 \kappa_{1}} \approx 81$ between magnetic material constants at which the coercivity is small. This generic formula serves as a theoretical guide to the alloy-development program, by suggesting alternative combinations of material constants-beyond $\kappa_{1}=$ 0 - to develop soft magnets. While this relation is based on $\langle 100\rangle$ easy axes and might differ for alloys with different easy axes, our work is intended to guide the search for soft magnetism in ordinary ferromagnets with large $m_{s}$. In future work, we intend to investigate other easy axes, elastic stiffness constants, to further lower coercivity in magnetic alloys ${ }^{35}$. With the recent advances in atomic-scale engineering, the compositions of magnetic alloys can be tuned atom-by-atom ${ }^{37,38}$. For these experimental approaches, our prediction of the material constant formula could serve as a guiding principle, to engineer magnetic alloy compositions to small hysteresis. Overall, the fundamental relationship between material constants provides initial steps to experimentalists to discover soft magnets with high magnetocrystalline anisotropy constants.

In conclusion, the present findings contribute to a more nuanced understanding of how material constants, such as magnetocrystalline anisotropy and magnetostriction constants, affect magnetic hysteresis. Specifically, magnetoelastic interactions have been regarded to play a negligible role in lowering magnetic coercivity. Given the current findings, we quantitatively demonstrate that the delicate balance between magnetocrystalline anisotropy, magnetostriction constants, and the spike-domain microstructure (localized disturbance) is necessary to lower magnetic coercivity. We propose a mathematical relationship between material constants $\frac{\left(c_{11}-c_{12}\right) \lambda_{100}^{2}}{2 \kappa_{1}} \approx 81$ at which minimum coercivity can be achieved in a material with $\langle 100\rangle$ easy axes. More generally, our findings serve as a theoretical guide to discover novel combinations of material constants that lower coercivity in magnetic alloys.

\section{METHODS}

\section{Micromagnetics}

In our coercivity tool, we use micromagnetics theory that describes the total free energy as a function of magnetization $\mathbf{m}$, strain $\mathbf{E}$, and magnetostatic field $\mathbf{H}_{\mathbf{d}}$ :

$$
\begin{aligned}
\psi= & \int_{\Omega}\left\{\nabla \mathbf{m} \cdot \mathbf{A m}+\kappa_{1}\left(\mathrm{~m}_{1}^{2} \mathrm{~m}_{2}^{2}+\mathrm{m}_{2}^{2} \mathrm{~m}_{3}^{2}+\mathrm{m}_{3}^{2} \mathrm{~m}_{1}^{2}\right)+\frac{1}{2}\left[\mathbf{E}-\mathbf{E}_{\mathbf{0}}(\mathbf{m})\right]\right. \\
& \left.\cdot \mathbb{C}\left[\mathbf{E}-\mathbf{E}_{\mathbf{0}}(\mathbf{m})\right]-\mu_{0} m_{s} \mathbf{H}_{\mathrm{ext}} \cdot \mathbf{m}\right\} \mathbf{d} \mathbf{x}+\int_{\mathbb{R}^{3}} \frac{\mu_{0}}{2}\left|\mathbf{H}_{\mathbf{d}}\right|^{2} \mathrm{~d} \mathbf{x} .
\end{aligned}
$$

The form of Eq. (10) is identical to that used in our previous work, in which we detail the meaning of the specific terms, material constants, and normalizations ${ }^{16}$. For the present work, we note that the exchange energy $\nabla \mathbf{m} \cdot A \nabla \mathbf{m}$ penalizes spatial gradients of magnetization. More generally, the anisotropy energy for cubic alloys would have contributions from higher order energy terms (e.g., $k_{2}\left(m_{1}^{2} m_{2}^{2} m_{3}^{2}\right)$ ), and these additional anisotropy coefficients are likely in general to contribute to our coercivity calculations. Although including these higher-order anisotropy coefficients, e.g., $\kappa_{2}$, could affect the easy axes of magnetization of the material. For example at $\kappa_{1} \approx \frac{-\kappa_{2}}{2}$ other crystallographic directions, such as [111], [110], and a family of irrational directions, would have similarly small magnetocrystalline anisotropy energy. This warrants a systematic investigation in a future study, especially with $\kappa_{1}$, $\kappa_{2}, K_{3}$ near actual measured values. However, we do not think that these higher order terms would significantly affect our coercivity calculations for the following reasons: first, in our computations, we model an oblate ellipsoid (pancake-shaped) that supports an in-plane magnetization. This ellipsoid geometry and the defect shape penalizes out-of-plane magnetization and thus $\mathrm{m}_{3} \approx 0$ in our calculations of the magnetic hysteresis. Consequently, the energy contribution from the higher-order anisotropy term, $\kappa_{2}\left(\mathrm{~m}_{1}^{2} \mathrm{~m}_{2}^{2} \mathrm{~m}_{3}^{2}\right)$, is negligible in our computations. Second, in the nondimensional form of the free energy (see Eq. (5)), the energy 
contribution from the higher-order anisotropy term would scale as $\frac{k_{2}}{k_{1}}\left(m_{1}^{2} m_{2}^{2} m_{3}^{2}\right)$ with $|\mathbf{m}|=1$. This sixth-order energy term is expected not to change the coercivity calculations significantly. We propose to investigate the precise role of the higher-order anisotropy terms in a future study, however, as a first step, we investigate coercivity as a function of magnetocrystalline anisotropy $\kappa_{1}$ and magnetostriction constant $\lambda_{100}$. The elastic energy $\frac{1}{2}\left[\mathbf{E}-\mathbf{E}_{\mathbf{0}}(\mathbf{m})\right] \cdot \mathbb{C}\left[\mathbf{E}-\mathbf{E}_{\mathbf{0}}(\mathbf{m})\right]$ penalizes mechanical deformation away from the preferred strains, and the external energy, $\mu_{0} \mathbf{H}_{\text {ext }} \cdot \mathbf{m}$ accounts for the mutual interaction between magnetization moment and the applied field. Finally, the magnetostatic energy $\frac{\mu_{0}}{2}\left|\mathbf{H}_{\mathbf{d}}\right|^{2}$ computed in all of space $\mathbb{R}^{3}$ penalizes the stray fields generated by the magnetic body in its surroundings.

We compute the evolution of the magnetization using an energyminimization technique, the generalized Landau-Lifshitz-Ginzburg equation, see Fig. 4:

$\frac{\partial \mathbf{m}}{\partial \tau}=-\mathbf{m} \times \mathcal{H}-a \mathbf{m} \times(\mathbf{m} \times \mathcal{H})$.
Here, $\mathcal{H}=-\frac{1}{\mu_{0} m_{s}^{2}} \frac{\delta \psi}{\delta m}$ is the effective field, $\tau=\gamma m_{s} t$ is the dimensionless time step, $\gamma$ is the gyromagnetic ratio, and $a$ is the damping constant. We numerically solve Eq. (11) using the Gauss-Siedel projection method ${ }^{18}$, and identify equilibrium states when the magnetization evolution converges, $\left|\mathbf{m}^{\mathrm{n}+1}-\mathbf{m}^{\mathrm{n}}\right|<10^{-9}$. At each iteration we compute the magnetostatic field $\mathbf{H}_{\mathbf{d}}=-\nabla \zeta_{\mathbf{m}}$ and the strain $\mathbf{E}$ by solving their respective equilibrium equations:

$\nabla \cdot\left(-\nabla \zeta_{\mathbf{m}}+m_{s} \mathbf{m}\right)=0 \quad$ on $\mathbb{R}^{3}$

$\nabla \cdot \mathbb{C}\left(\mathbf{E}-\mathbf{E}_{0}\right)=0 \quad$ on $\mathcal{E}$.

The magnetostatic equilibrium condition arises from the Maxwell equations, namely $\nabla \times \mathbf{H}_{\mathbf{d}}=0 \rightarrow \mathbf{H}_{\mathbf{d}}=-\nabla \zeta_{\mathbf{m}}$ and $\nabla \cdot \mathbf{B}=\nabla \cdot\left(\mathbf{H}_{\mathbf{d}}+m_{s} \mathbf{m}\right)=0$.

In our calculations, we model a finite-sized domain $\Omega$ centered around a nonmagnetic defect $\Omega_{d}$, see inset images in Fig. 4. This domain is several times smaller than the actual size of the ellipsoid $\mathcal{E}$, see Fig. 1. We define the total demagnetization field as a sum of the local $\widetilde{\mathbf{H}}(\mathbf{x})$ and nonlocal

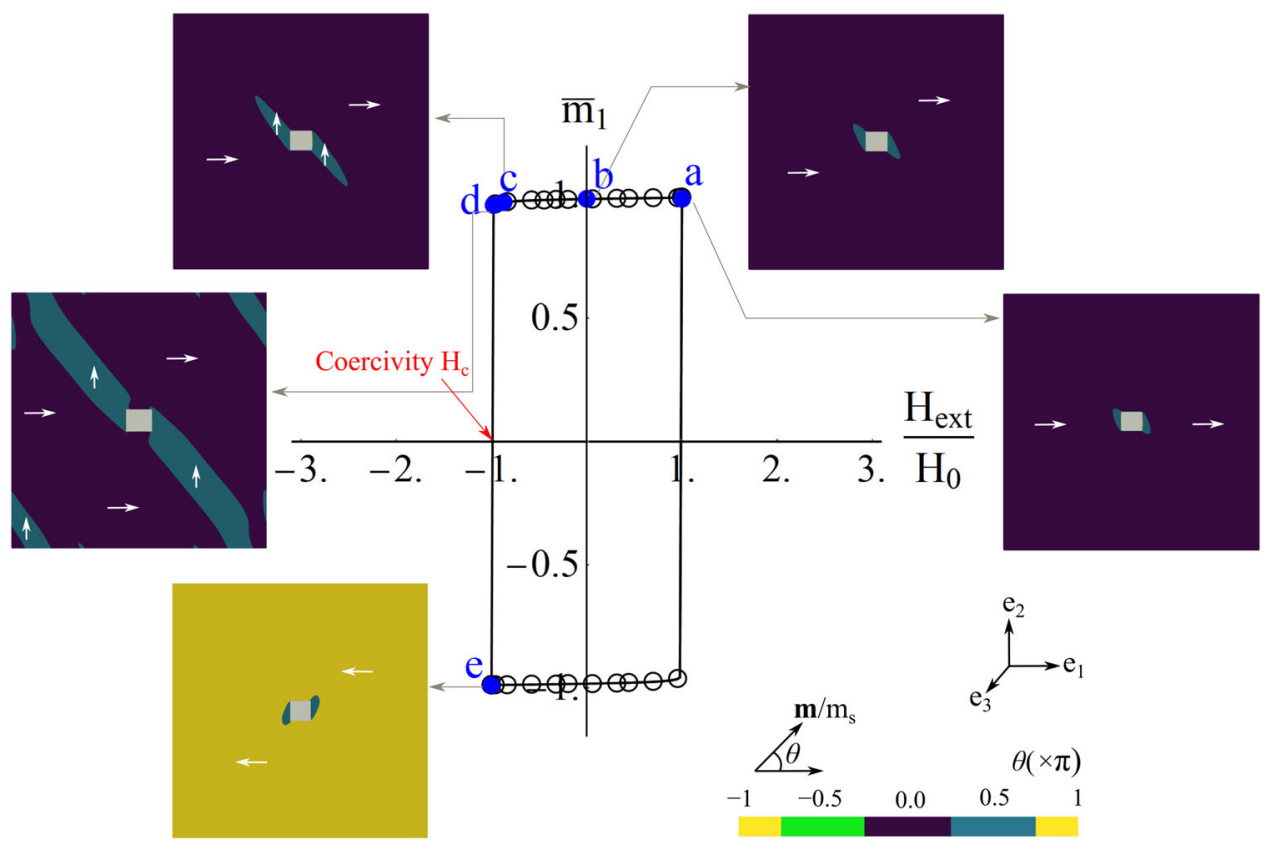

Fig. 4 Growth of a spike-domain microstructure during a typical magnetization reversal. A spike domain forms around a nonmagnetic inclusion in the computation domain. This spike grows gradually on decreasing the external field (a-c). At the coercivity value, the spike domain grows abruptly, resulting in magnetization reversal $(\mathbf{d}, \mathbf{e})$. The hysteresis loop plots the average magnetization in the computation domain $\overline{\mathrm{m}}_{1}$ as a function of the applied field $\mathrm{H}_{\mathrm{ext}}$.

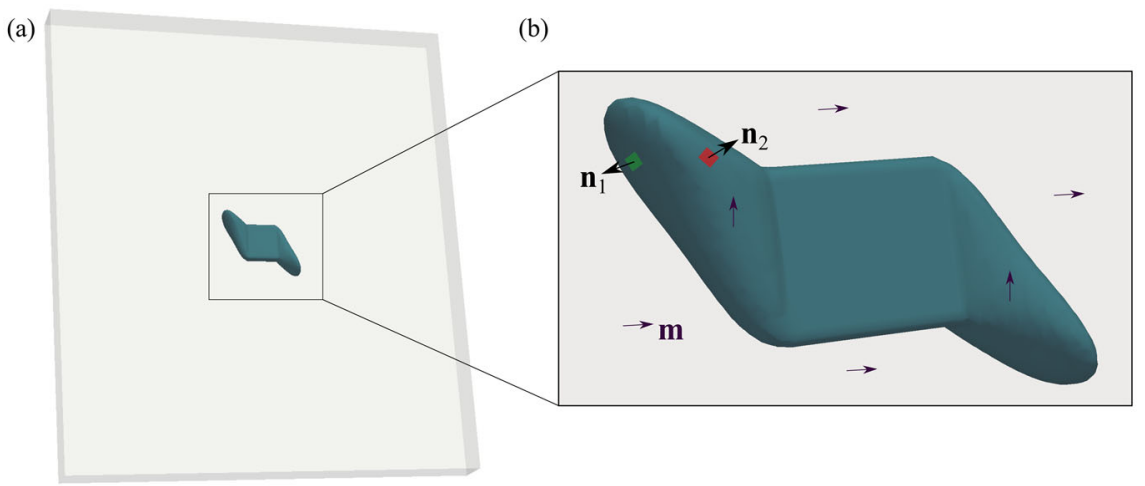

Fig. 5 A 3D visualization of the needle domain. a This needle domain forms around an embedded defect in our micromagnetic computations. b The inset figure shows the needle domain with distinct surfaces (marked by surface-normal vectors $\mathbf{n}_{1}, \mathbf{n}_{2}$ ). The surface $\mathbf{n}_{1}$ approximately satisfies the strain-compatibility condition, however, the surface $\mathbf{n}_{2}$ is not compatible and contributes to nonzero elastic energy in the system. The magnetization is denoted by $\mathbf{m}$. 


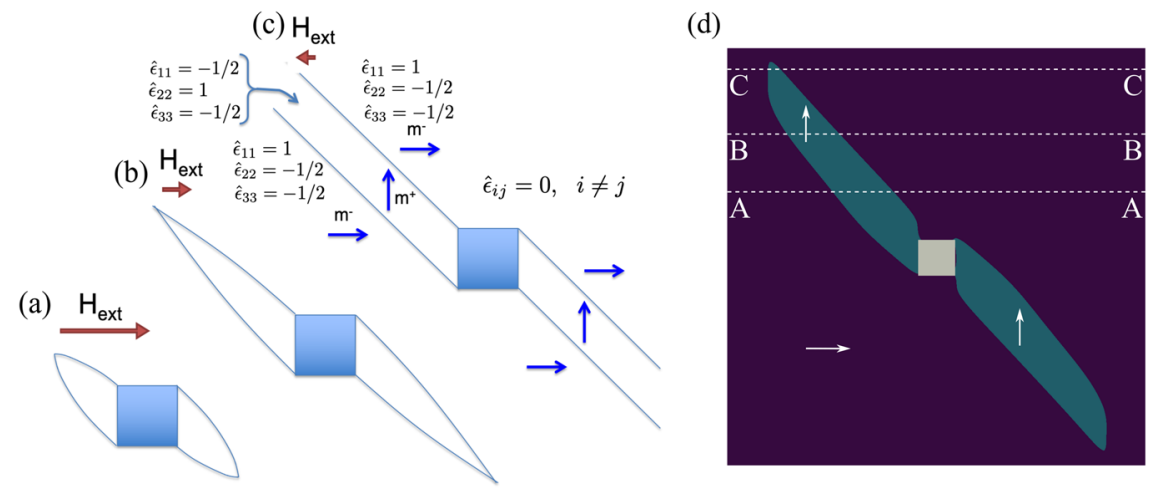

Fig. 6 Schematic of the metastability of the Néel spikes. a With large applied field the spikes are collapsed onto the nonmagnetic defect, stabilized by (diffuse) domain wall and demagnetization energy. b As the applied field is decreased, the spikes grow, so as to decrease magnetostrictive and demagnetization energies. c The associated energy barrier is breached at small negative applied fields, and the spikes run to the boundary. The state (c) is transient, and precedes the full switch to the lower energy [100] magnetization; the final state is like (a) except the spike domains are pointing NE and SW (see Fig. 4e). d An example simulation of the spike domain at its transient domain state during magnetization reversal. Labels "AA", "BB", and " $C C$ " mark lengths across which we examine the strain distribution in Fig. 7.

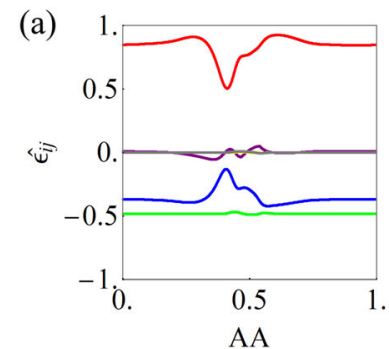

(b)
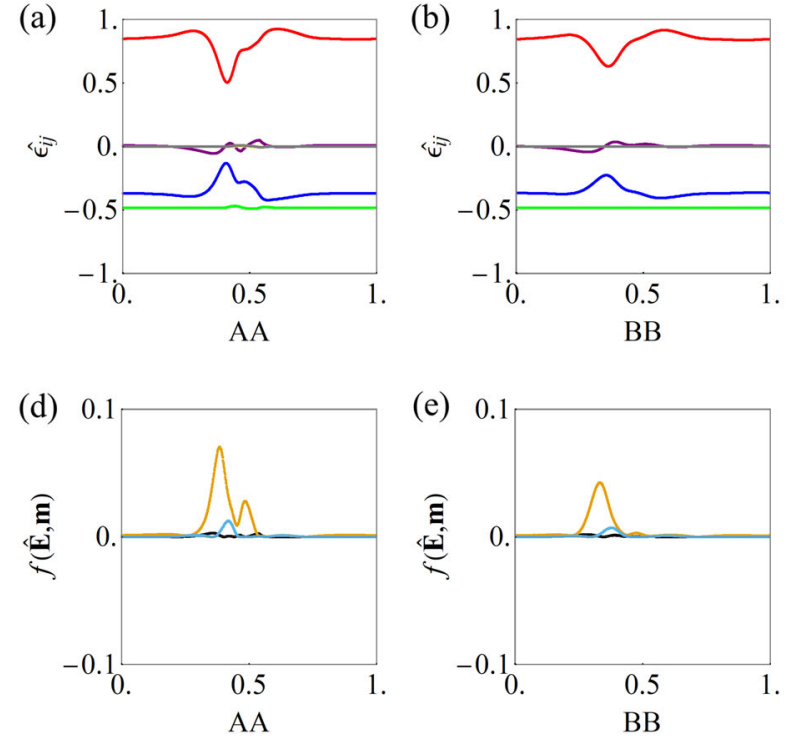
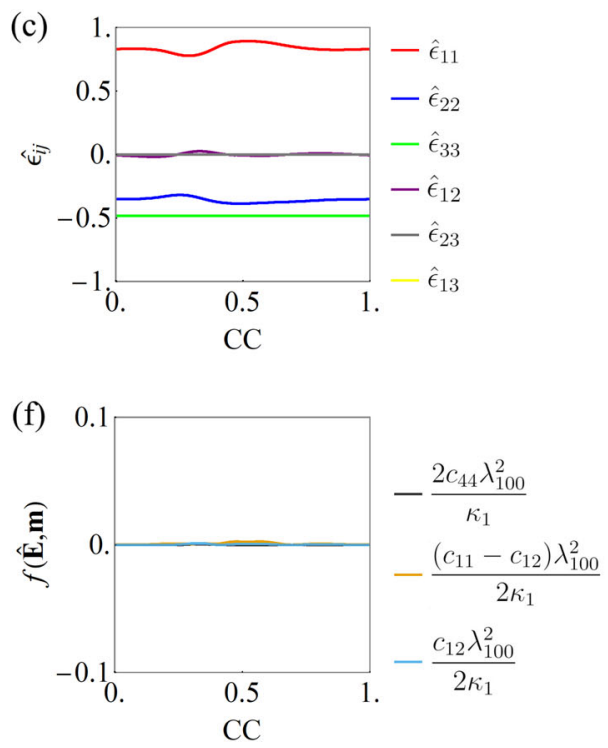

Fig. 7 The strain and elastic energy distribution across the needle domain. a-c The strain distribution $\hat{\epsilon}_{i j}$ across lengths " $A A^{\prime \prime}$, "BB", and " $C C$ " (as marked in Fig. $6(\mathrm{~d})$ ) in the computational domain. The strain distribution from the simulation, $\hat{\epsilon}_{11} \approx 1, \hat{\epsilon}_{22} \approx \hat{\epsilon}_{33} \approx-0.5$, is consistent with the theoretical analysis in Fig. 6(a). The strains at the domain walls near the spike domain vary, indicating the diffuseness of the walls. The shear strains are mostly zero throughout the computational domain. (d-f) A plot of the three polynomials $f(\hat{\mathbf{E}}, \mathbf{m})$ that accompany coefficients $\frac{2 c_{44} \lambda_{100}^{2}}{\kappa_{1}}, \frac{\left(c_{11}-c_{12}\right) \lambda_{100}^{2}}{2 \kappa_{1}}$, and $\frac{c_{12} \lambda_{100}^{2}}{2 \kappa_{1}}$ in Eq. (5). In line with our hypothesis the polynomials accompanying $\frac{2 c_{44} \lambda_{100}^{2}}{\kappa_{1}}$ and $\frac{c_{12} \lambda_{100}^{2}}{2 \kappa_{1}}$ make negligible energy contributions for magnetization reversal at the transient state of the spike domain, and the polynomial accompanying $\frac{\left(c_{11}-c_{12}\right) \lambda_{100}^{2}}{2 \kappa_{1}}$ serves as an energy barrier for magnetization reversal at the transient domain state.

contributions $\overline{\mathbf{H}}$. The local contribution $\widetilde{\mathbf{H}}(\mathbf{x})$ varies spatially and accounts for the magnetostatic fields generated from defects and other imperfections inside the body. We calculate this local contribution by solving $\nabla$. $\left(\widetilde{\mathbf{H}}+m_{s} \widetilde{\mathbf{m}}\right)=0$ on $\Omega$. The nonlocal contribution is computed as $\overline{\mathbf{H}}=-\mathbf{N} m_{s} \overline{\mathbf{m}}$. Here, $\mathbf{N}$ is the demagnetization-factor matrix that is a tabulated geometric property of the ellipsoid. We note, from the tabulated values in ref. ${ }^{39}$, the demagnetization factors for an oblate ellipsoid (pancake-shaped) are $\mathrm{N}_{11}=\mathrm{N}_{22}=0, \mathrm{~N}_{33}=1$. The $\overline{\mathbf{m}}$ is the constant magnetization that is defined, such that $\int_{\Omega} \mathbf{m}(\mathbf{x}) \mathrm{d} \mathbf{x}=0$. This decomposition simplifies our computational complexity, because we now model a local domain $\Omega$ that is much smaller than modeling a domain in $\mathbb{R}^{3}$, and yet account for the demagnetization contributions from both the body geometry and the local defects. This decomposition is justified in the appendix of our previous paper ${ }^{16}$. Both the magnetostatic and mechanical equilibrium conditions in Eqs. (12)-(13) are solved in Fourier space, see refs. ${ }^{16,19}$ for further details.

\section{Numerical calculations}

In the present work, we calibrate our micromagnetic model for the FeNi alloy with the following material constants: $A=10^{-11} \mathrm{~J} \mathrm{~m}^{-1}, m_{s}=10^{6} \mathrm{Am}^{-2}$, $\mu_{0}=1.3 \times 10^{-6} \mathrm{NA}^{-2}, c_{11}=240.8 \mathrm{GPa}, c_{12}=89.2 \mathrm{GPa}$, and $c_{44}=75.8 \mathrm{GPa}$. The values of $\kappa_{1}, \lambda_{100}, \lambda_{111}$ are systematically varied as detailed in the "Results" section. Here, note that magnetic alloys with positive and negative magnetocrystalline anisotropy constants have their easy axes along $\langle 100\rangle$ and $\langle 111\rangle$ crystallographic directions, respectively. To accommodate this change of easy axes, we transform the energy potential from a cubic basis, and further details of this transformation are described in the appendix of ${ }^{16}$.

We compute the coercivity of magnetic ellipsoids as a function of the magnetocrystalline anisotropy and magnetostriction material constants. In each computation, we model a domain of size $64 \times 64 \times 24$ containing a defect with $16 \times 16 \times 6$ grid points. We choose a grid size such that the domain walls span across 3-4 unit cells. We initialize the computational domain with a uniform magnetization $\mathbf{m}=\mathrm{m}_{1}$, and force the 
magnetization inside the defect to be zero throughout the computation, $|\mathbf{m}|=0$, see Fig. 4. We apply a large external field along the easy axes, $\mathbf{H}_{\text {ext }}$ $>0$, and decrease it gradually in steps of $\delta \mathbf{H}=0.25 \mathbf{e}_{1}$ Oe. As we decrease the applied field, a spike domain forms around the defect, see Fig. 1. This spike domain grows in size as the applied field is further lowered until a critical field value-known as the coercivity $\mathrm{H}_{\mathrm{c}}$-at which the magnetization vector reverses. We use this approach to predict the coercivity of the magnetic alloys at each combination of the magnetocrystalline anisotropy and magnetostriction constants.

Specifically, in Study 1 and Study 2 we carry out a total of $n=2163$ and $n=605$ computations respectively. In these computations, we systematically vary the material constants in the parameter space range of $0 \leq$ $\kappa_{1} \leq 2000 \mathrm{~J} \mathrm{~m}^{-3},-2000 \mu \epsilon \leq \lambda_{100} \leq 2000 \mu \epsilon$, and $0 \leq \lambda_{111} \leq 600 \mu \epsilon$, respectively. Our investigation shows that the minimum coercivity is attained for a parabolic relation $\kappa_{1} \propto \lambda_{100}^{2}$, and a total of over 2500 computations are necessary to confirm this relationship.

\section{DATA AVAILABILITY}

The authors declare that the data supporting the findings of this study are available within the paper and its supplementary information files. Furthermore, additional data that support the findings of this study are available from the corresponding author upon request.

Received: 5 August 2021; Accepted: 26 November 2021; Published online: 13 January 2022

\section{REFERENCES}

1. Fert, A. Nobel Lecture: Origin, development, and future of spintronics. Rev. Mod. Phys. 80, 1517 (2008)

2. Silveyra, J. M., Ferrara, E., Huber, D. L., \& Monson, T. C. Soft magnetic materials for a sustainable and electrified world. Science, 362, aao0195 (2018).

3. Teter, J. P., Clark, A. E., Wun-Fogle, M. \& McMasters, O. D. Magnetostriction and hysteresis for $\mathrm{Mn}$ substitutions in $\left(\mathrm{Tb}_{x} \mathrm{Dy}_{1-x}\right)\left(\mathrm{Mn}_{y} \mathrm{Fe}_{1-y}\right)_{1.95}$. IEEE Trans. Magn. 26, 1748-1750 (1990).

4. Clark, A. E., Teter, J. P. \& McMasters, O. D. Magnetostriction "jumps" in twinned $\mathrm{Tb}_{0.3} \mathrm{Dy}_{0.7} \mathrm{Fe}_{1.9}$. J. Appl. Phys. 63, 3910-3912 (1988)

5. Yang, S. et al. Large magnetostriction from morphotropic phase boundary in ferromagnets. Phys. Rev. Lett. 104, 197201 (2010)

6. Bergstrom, R.Jr. et al. Morphotropic phase boundaries in ferromagnets: $\mathrm{Tb}_{1}$ ${ }_{x} \mathrm{Dy}_{x} \mathrm{Fe}_{2}$ Alloys. Phys. Rev. Lett. 111, 017203 (2013).

7. $\mathrm{Hu}, \mathrm{C}$. C. et al. Room-temperature ultrasensitive magnetoelastic responses near the magnetic-ordering tricritical region. J. Appl. Phys. 130, 063901 (2021).

8. Bozorth, R. M. The permalloy problem. Rev. Mod. Phys. 25, 42 (1953).

9. Takahashi, M., Nishimaki, S. \& Wakiyama, T. Magnetocrystalline anisotropy and magnetostriction of Fe-Si-Al (Sendust) single crystals. J. Magn. Magn. Mater. 66 55-62 (1987)

10. Tickle, R. \& James, R. D. Magnetic and magnetomechanical properties of $\mathrm{Ni}_{2} \mathrm{MnGa}$. J. Magn. Magn. Mater. 195, 627-638 (1999).

11. Atulasimha, J. \& Flatau, A. B. A review of magnetostrictive iron-gallium alloys. Smart Mater. Struct. 20, 043001, (2011).

12. Clark, A. E., Wun-Fogle, M., Restorff, J. B. \& Lograsso, T. A. Magnetostrictive properties of Galfenol alloys under compressive stress. Mater. Trans. 43, 881-886 (2002).

13. Brown Jr, W. F. Micromagnetics 18 (Interscience Publishers, 1963).

14. Aharoni, A. \& Shtrikman, S. Magnetization curve of the infinite cylinder. Phys. Rev. 109, 1522 (1958).

15. Brown, W. F. Magnetostatic Principles in Ferromagnetism (North-Holland Publishing Company, 1962).

16. Renuka Balakrishna, A. \& James, R. D. A tool to predict coercivity in magnetic materials. Acta Mater. 208, 116697 (2021).

17. Renuka Balakrishna, A. \& James, R. D. A solution to the permalloy problem-A micromagnetic analysis with magnetostriction. Appl. Phys. Lett. 118, 212404 (2021).

18. Wang, X. P., Garcia-Cervera, C. J. \& Weinan, E. A Gauss-Siedel projection method for micromagnetics simulations. J. Comput. Phys. 171, 357-372 (2001).

19. Zhang, J. X. \& Chen, L. Q. Phase-field microelasticity theory and micromagnetic simulations of domain structures in giant magnetostrictive materials. Acta Mater. 53, 2845-2855, (2005)

20. Wang, J. \& Zhang, J. A real-space phase field model for the domain evolution of ferromagnetic materials. Int. J. Solids and Struct. 50, 3597-3609 (2013).
21. Hubert, A., \& Schäfer, R. Magnetic domains: the analysis of magnetic microstructures (Springer Science \& Business Media, 2008).

22. Otto, F. \& Viehmann, T. Domain branching in uniaxial ferromagnets: asymptotic behavior of the energy. Calc. Var. Partial Differ. Equ. 38, 135-181 (2010).

23. Otto, F. \& Steiner, J. The concertina pattern. Calc. Var. Partial Differ. Equ. 39, 139-181 (2010)

24. Döring, L., Ignat, R. \& Otto, F. A reduced model for domain walls in soft ferromagnetic films at the cross-over from symmetric to asymmetric wall types. J. Eur. Math. Soc. 16, 1377-1422 (2014).

25. Cinti, E. \& Otto, F. Interpolation inequalities in pattern formation. J. Funct. Anal. 271, 3348-3392 (2016)

26. Brown, W. F. Magnetoelastic interactions (Springer, 1966).

27. Pilet, N.The relation between magnetic hysteresis and the micromagnetic state explored by quantitative magnetic force microscopy, Doctoral dissertation (University of Basel, 2006).

28. Knüpfer, H., Kohn, R. V. \& Otto, F. Nucleation barriers for the cubic-to-tetragonal phase transformation. Commun. Pure Appl. Math. 66, 867-904 (2013).

29. Zhang, Z., James, R. D. \& Müller, S. Energy barriers and hysteresis in martensitic phase transformations. Acta Mater. 57, 4332-4352 (2009).

30. Zwicknagl, B. Microstructures in low-hysteresis shape memory alloys: scaling regimes and optimal needle shapes. Arch. Ration. Mecha. Anal. 213, 355-421 (2014).

31. Cui, J. et al. Combinatorial search of thermoelastic shape-memory alloys with extremely small hysteresis width. Nat. Mater. 5, 286-290 (2006).

32. Thomas, S. V. Nanocrystallites via direct melt spinning of $\mathrm{Fe}_{77} \mathrm{Ni}_{5.5} \mathrm{Co}_{5.5} \mathrm{Zr}_{7} \mathrm{~B}_{4} \mathrm{Cu}$ for enhanced magnetic softness. Phys. Status Solidi 217, 1900680 (2020).

33. McHenry, M. E., Willard, M. A. \& Laughlin, D. E. Amorphous and nanocrystalline materials for applications as soft magnets. Prog. Materi. Sci. 44, 291-433 (1999).

34. Soldatov, I., Andrei, P. \& Schaefer, R. Inverted hysteresis, magnetic domains, and hysterons. IEEE Magn. Lett. 11, 1-5 (2020).

35. Ahani, N., Daware, A. \& Renuka Balakrishna, A. In preparation (2021).

36. James, R. D. \& Hane, K. F. Martensitic transformations and shape-memory materials. Acta Mater. 48, 197-222 (2000).

37. Ouazi, S. et al. Atomic-scale engineering of magnetic anisotropy of nanostructures through interfaces and interlines. Nat. Commun. 3, 1-9, (2012).

38. Kablov, E. N. et al. Bifurcation of magnetic anisotropy caused by small addition of $\mathrm{Sm}$ in $\left(\mathrm{Nd}_{1-x} \mathrm{Sm}_{x} \mathrm{Dy}\right)(\mathrm{FeCo}) \mathrm{B}$ magnetic alloy. J. Appl. Phys. 117, 243903 (2015).

39. Osborn, J. A. Demagnetizing factors of the general ellipsoid. Phys. Rev. 67, 351 (1945).

40. Schäfer, R. \& Schinnerling, S. Tomography of basic magnetic domain patterns in ironlike bulk material. Phys. Rev. B 101, 214430 (2020).

\section{ACKNOWLEDGEMENTS}

The authors acknowledge the Center for Advanced Research Computing at the University of Southern California and the Minnesota Supercomputing Institute at the University of Minnesota for providing resources that contributed to the research results reported within this paper. The authors would like to thank Anjanroop Singh (University of Minnesota) for help in checking some of the calculations. A.R.B acknowledges the support of a Provost Assistant Professor Fellowship, Gabilan WiSE fellowship, and USC's start-up funds. R.D.J acknowledges the support of a Vannevar Bush Faculty Fellowship. The authors thank NSF (DMREF-1629026) and ONR (N0001418-1-2766) for partial support of this work.

\section{AUTHOR CONTRIBUTIONS}

A.R.B. and R.D.J. conceptualized the project, designed the methodology, and procured funding. A.R.B. worked on model development, theoretical analysis, and visualization of data. R.D.J. worked on the theoretical calculations. Both authors were involved with the writing of the paper.

\section{COMPETING INTERESTS}

The authors declare no competing interests.

\section{ADDITIONAL INFORMATION}

Supplementary information The online version contains supplementary material available at https://doi.org/10.1038/s41524-021-00682-7.

Correspondence and requests for materials should be addressed to Ananya Renuka Balakrishna. 
Reprints and permission information is available at http://www.nature.com/ reprints

Publisher's note Springer Nature remains neutral with regard to jurisdictional claims in published maps and institutional affiliations.

Open Access This article is licensed under a Creative Commons Acc Attribution 4.0 International License, which permits use, sharing,
adaptation, distribution and reproduction in any medium or format, as long as you give appropriate credit to the original author(s) and the source, provide a link to the Creative Commons license, and indicate if changes were made. The images or other third party material in this article are included in the article's Creative Commons license, unless indicated otherwise in a credit line to the material. If material is not included in the article's Creative Commons license and your intended use is not permitted by statutory regulation or exceeds the permitted use, you will need to obtain permission directly from the copyright holder. To view a copy of this license, visit http://creativecommons. org/licenses/by/4.0/.

(c) The Author(s) 2022 